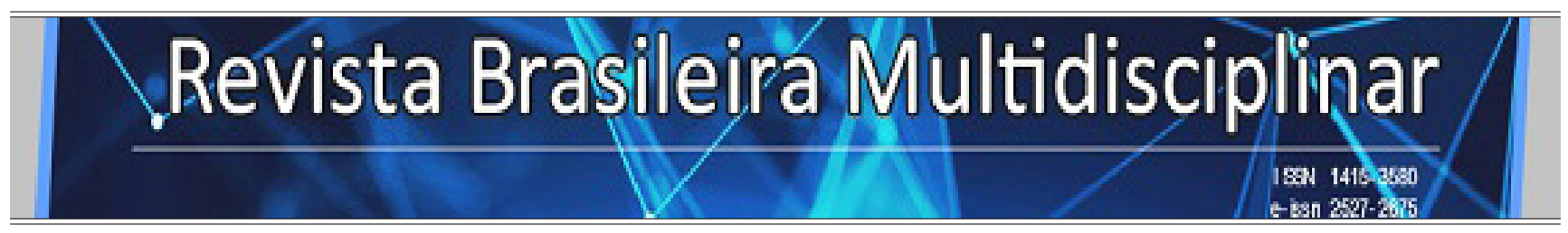

http://revistarebram.com/index.php/revistauniara

\title{
QUALIDADE DE VIDA DE MULHERES NA ENGENHARIA CIVIL
}

Caroline Urias Challouts*; Tânia Maria Gomes da Silva ${ }^{\star *}$; Natália Quevedo dos Santos***.

* Graduanda em Engenharia Civil, Mestranda no Programa de Pós-Graduação em Promoção da Saúde Universidade Unicesumar. Maringá. Paraná.

** Doutora em História, Docente do Programa de Pós-Graduação em Promoção da Saúde da Universidade Unicesumar.

*** Graduada em Fisioterapia. Mestranda no Programa de Pós-Graduação em Promoção da Saúde pela Universidade Unicesumar.

*Autor para correspondência e-mail: carolineuchallouts@gmail.com

\section{Palavras-chave}

Engenharia Civil

Qualidade de Vida

Saúde da Mulher

\section{KEYWORDS}

Civil Engineering

Quality of Life

Women's Health
Resumo: No Brasil, o número de mulheres na área da engenharia civil aumentou consideravelmente nas últimas décadas. Contudo, não há igual relação de crescimento nos cargos de chefia feminina. Acredita-se que esse baixo número se dá por conta dos estereótipos de gênero ainda presentes na nossa sociedade, colocando as engenheiras em uma situação de vulnerabilidade. Considerando que viver situações de inequidade de qualquer natureza pode ter impactos na vida das pessoas, o presente estudo teve como objetivo verificar o nível de qualidade de vida das engenheiras civis associadas ao CREA-SP. Fundamentou- se na aplicação de questionário abreviado WHOQOL-bref. Os dados foram analisados pelo software Excel Microsoft Corporation, apresentados por meio de gráficos e tabelas. Os resultados apontaram não haver uma relação direta entre inequidades de gênero e qualidade de vida. A pesquisa empírica evidenciou que as entrevistadas apresentavam boa qualidade de vida (627\%), com o domínio psicológico apresentando menor escore $(58,12 \%)$. Evidencia-se a necessidade de mais estudos que avaliam a qualidade de vida de mulheres na engenharia civil como em outras áreas, para proporcionar mais estratégias para o autocuidado e promoção da saúde para essa população.

\section{QUALITY OF LIFE WOMEN IN CIVIL ENGINEERING}

In Brazil, the number of women in the field of civil engineering has increased considerably in recent decades. However, there is not equal growth in female leadership positions. It is believed that this low number is due to the gender stereotypes still present in our society, putting female engineers in a situation of vulnerability. Considering that living in situations of inequity of any nature can have an impact on people's lives, this study aimed to verify the level of quality of life of civil engineers associated with CREA-SP. It was based on the application of a WHOQOL-bref abbreviated questionnaire. The data were analyzed by Excel Microsoft Corporation software, presented through graphs and tables. The results showed no direct relationship between gender inequalities and quality of life. The empirical research showed that the women interviewed had good quality of life (627\%), with the psychological domain presenting a lower score (58.12\%). It is evident the need for more studies that evaluate the quality of life of women in civil engineering as in other areas, to provide more strategies for self-care and health promotion for this population. 


\section{INTRODUÇÃo}

A área das engenharias sempre foi majoritariamente masculina. Essa situação vem apresentando nítida mudança. No Brasil, entre 2003 e 2013, houve um crescimento de 132,2\% no número de engenheiras, que passou de 24.554 para 57.022; índice bem superior ao masculino, de 78,3\% (DIAS, 2016). Em 1930, havia doze engenheiras registradas no Conselho Regional de Engenharia e Agronomia (CREA); em 2018 esse número chegou a 1.444.343, incluindo profissionais na ativa ou não (CONFEA, 2020). Conquanto seja animador, esse crescimento não deve ser comemorado sem uma análise apurada, pois não há relação direta entre os dados do CREA e o pleno reconhecimento das engenheiras no exercício da profissão. Ressalte-se, como exemplo, que a função de presidente do CONFEA/CREA, órgão que existe desde 1996, foi ocupada por uma mulher somente uma única vez (CONFEA, 2020), evidenciando que os espaços de liderança ainda são ocupados majoritariamente pelos homens (CHALLOUTS et al., 2019; LOMBARDI, 2017).

Defende-se que o reduzido número de mulheres em cargos de comando é consequência de estereótipos de gênero, perceptível em distintas profissões, mas agravadas em algumas, como as engenharias. Esses estereótipos ajudam a cristalizar papéis de gênero, relegando as mulheres ao desempenho de profissões voltadas ao cuidado com o outro, tais como enfermeiras, assistentes sociais e professoras, enquanto os homes são vinculados às profissões que exigem força e capacidade de comando (LIBERATO; ANDRADE, 2018). Embora no mundo real essa situação tenha sido há muito desconstruída, as representações sociais continuam legitimando as distinções sexuais de gênero. A divisão sexual do trabalho (KERGOAT, 2009), conquanto atenuada, não deixou de existir.

O conceito de gênero surgiu na década de 1970, nos Estados Unidos. Uma década depois estava plenamente incorporado aos estudos interdisciplinares, entendido como uma ferramenta analítica importante para separar definitivamente elementos culturais e históricos (gênero) daqueles marcados pela biologia (sexo). Assim, gênero desnaturaliza o ser homem e ser mulher (SCOTT, 1995). Segundo Matos (2015), os comportamentos masculinos e femininos frente a diferentes situações sociais vêm sendo compreendidos como fruto de um intenso e duradouro processo sociocultural de gênero. Para a autora, há sempre alguma expectativa sobre como se deve andar, mostrar o corpo, se sentar, falar, comer, amar, fazer política, etc, moldado pelo gênero e quem se afasta dessa normatividade recebe críticas e sofre preconceitos. Concordamos com Matos (2015) e acrescentamos que as pessoas também escolhem suas profissões de forma gendrada, isto é, as definições culturais sobre gênero são elementos que condicionam a distribuição de mulheres e homens na estrutura ocupacional da sociedade, "assim como suas chances de desenhar determinadas trajetórias ocupacionais e a desigualdade de renda” (PICANÇO, 2005, p. 150). Um estudo de Lombardi (2017) afirma que, à época, enquanto 57\% dos engenheiros ganhavam mais de dez salários mínimos e $17 \%$ mais de 20 salários mínimos, apenas $44 \%$ das engenheiras recebiam essa remuneração.

A relação entre equidade e saúde sempre foi observada nos estudos epidemiológicos, mas só no final da década de 1990 os profissionais de saúde tiveram maior preocupação "em analisar a distribuição das doenças a partir do reconhecimento de iniquidades sociais” (CHIESA et al., p. 289). Nas últimas décadas, vários estudos têm se voltado a entender as experiências das pessoas levando em conta os pertencimentos de gênero, raça/etnia, classe social, entre outros constituintes das relações humanas (SCHWARCZ, 2019; FARO; PEREIRA, 2011; LAGES et al., 2017; MASSIGNAN et al., 2019), visando entender como eles influenciam nos modos de viver, adoecer e até mesmo morrer (SILVA et al., 2020). Assim, profissionais da área da saúde têm se preocupado cada vez mais com os determinantes sociais da saúde, tidos agora como cientificamente apropriados (MAKSUD, 2014). Relações desiguais de gênero contribuem para colocar as mulheres em locais de menor reconhecimento profissional, podendo impactar na saúde delas, visto que existe uma relação direta entre discriminação e saúde (RODRIGUES, 2015). As pessoas vítimas de preconceito tendem à aquisição de hábitos prejudiciais à saúde, como consumo abusivo de álcool, cigarro e outras drogas; além de menor adesão em atividades promotoras de saúde, como a prática de atividades 
físicas e alimentação saudável (MATOS, 2015).

Articular uma relação entre equidade de gênero e as condições de vida e de saúde da mulher pressupõe um olhar ampliado sobre o binômio saúde/adoecimento, que não deve ser circunscrito ao modelo biomédico, mas estar atrelado ao modelo biopsicossocial, que leva em conta as condições concretas de vida dos sujeitos. A Carta de Ottawa já enfatizava a necessidade de uma visão mais holística para se pensar o conceito de saúde (OMS, 1986) e o mesmo faz, no Brasil, a Política Nacional de Promoção da Saúde (BRASIL/PNPS, 2014). Entre os seus temas transversais, a PNPS propõe o respeito às diversidades e diferenças de gênero.

Considerando que por estarem inseridas em uma área majoritariamente masculina, as engenheiras civis ainda enfrentam em sua vida profissional barreiras e estereótipos marcados por construções de gênero (CHALLOUTS et al., 2019), sem contar que viver situações de inequidade de qualquer natureza pode ter impactos na vida das pessoas, diante disso o presente estudo teve como objetivo verificar o nível de qualidade de vida das engenheiras civis.

\section{MATERIAL E MÉTODOS}

Trata-se de uma pesquisa transversal e com abordagem quantitativa. Os dados foram coletados através da aplicação on-line da versão abreviada e transcrita em português do questionário WHOQOL-bref Instrumento de Avaliação de Qualidade de vida da Organização Mundial da Saúde. O questionário ficou disponível durante o mês de outubro de 2019 através da plataforma on-line do Google Forms (ferramenta para a confecção de formulários on-line) e foi enviada pelo CREA-SP às engenheiras civis associadas do Estado de São Paulo, sendo excluídas aquelas associadas aos conselhos regionais de outros Estados ou não associadas a nenhum conselho.

O questionário WHOQOL-bref foi aplicado para avaliar a qualidade de vida das engenheiras civis. $\mathrm{O}$ instrumento continha 26 perguntas, sendo duas questões gerais sobre qualidade de vida e 24 questões são divididas em quatro domínios: físico, psicológico, social, e meio ambiente. O questionário utilizou a escala do tipo likert de cinco pontos e apresenta escore de zero a 100, sendo zero o pior nível e $100 \mathrm{o}$ melhor nível de qualidade de vida (FLECK et al., 1999).

Para realização da análise dos dados, foram calculados os escores e a estatística descritiva do questionário, por meio da utilização do software Microsoft Office Excel, conforme a ferramenta desenvolvida por Pedroso, Pilatti e Reis (2010). Essa ferramenta elimina os questionários que possuem mais de seis questões não respondidas ou com respostas inválidas e apresenta as médias de acordo com seus domínios, obtendo os mesmos resultados que no software SPSS.

A pesquisa foi apresentada ao Comitê de Ética do Centro Universitário de Maringá (UniCesumar), tendo recebido aprovação em setembro de 2019, sob o número 3.614.863.

\section{RESUlTAdos E discussão}

Após verificar como a desigualdade de gênero ainda é uma barreira a ser enfrentada pelas engenheiras civis, discutiremos a seguir como se encontra o nível de qualidade de vida destas mulheres $(n=15)$. A literatura é escassa no assunto de qualidade de vida em relação a mulheres que são engenheiras civis, portanto a qualidade de vida será vista de forma geral em mulheres que possuem atividades laborais.

Não há um consenso sobre o entendimento do termo qualidade de vida. O conceito é variável, e segundo estudos realizados por Day e Jankey (1996 apud PEREIRA et al., 2012), tem sido abordado a partir de quatro distintas variáveis: econômica, psicológica, biomédica ou holística. A primeira toma os indicadores sociais como elementos principais; a abordagem psicológica busca os indicadores relacionados às reações subjetivas dos indivíduos às suas vivências, estando ligada à felicidade e satisfação; na abordagem médica, a qualidade de vida é entendida na questão de oferecer melhorias nas condições de 
saúde e a capacidade de se viver plenamente e, por fim, as abordagens holísticas baseiam-se no entendimento de que o conceito de qualidade de vida é "multidimensional, apresenta uma organização complexa e dinâmica de seus componentes, difere de pessoa para pessoa de acordo com seu ambiente/contexto e mesmo entre duas pessoas inseridas em um contexto similar" (PEREIRA et al., 2012, p. 243). Percebe-se que o conceito é amplo e que não se relaciona apenas ao campo da saúde.

Tomaremos nesse trabalho a seguinte definição de qualidade de vida:

É considerada como a percepção do indivíduo de sua posição na vida no contexto da cultura e sistema de valores nos quais vive e em relação aos seus objetivos, expectativas, padrões e preocupações (WHOQOL, 1993).

No estudo de Marcacine e colaboradores (2019), foi verificada a qualidade de vida pelo instrumento WHOQOL-bref em 15 profissões entre mulheres, e foi possível observar que a média de cada domínio foi acima de 6,0 os que diferem do presente estudo, o que pode explicar esta diferença são as profissões, enquanto no estudo de Marcacine e colaboradores as profissões são consideradas como mais femininas e a do presente estudo na área da engenharia civil onde é caracterizada como uma profissão mais masculina mostrando portanto que ainda há necessidades de melhoras nestas profissões que são caracterizas mais masculinas (CERIBELI; ROCHA; PEREIRA, 2017).

Os resultados do presente estudo demonstram que a média de qualidade de vida no geral foi de $62,76 \%$, apresentando, assim, um resultado razoável. É importante salientar que o domínio que apresentou pior qualidade de vida foi o psicológico (58,61\%), sendo explicado pelo motivo que ainda as mulheres na área da engenharia civil apresentam uma satisfação parcial em relação a sua profissão, apresentando diferenças salariais e dificuldade na ascensão de carreira, bem como, a dupla jornada de trabalho com serviços domésticos e afazeres como esposa e mãe (OLIVEIRA et al., 2020). Analisando de forma mais apurada a (Q25) a respeito da frequência de sentimentos negativos, tais como mau humor, desespero, ansiedade e depressão, por parte das engenheiras, as respostas apontam que 6,7\% passam por isso muito frequentemente; $33,3 \%$, frequentemente; $26,7 \%$, sempre; $26,7 \%$, algumas vezes; e o restante nunca passou por isso.

Gráfico 01- Média geral dos domínios.

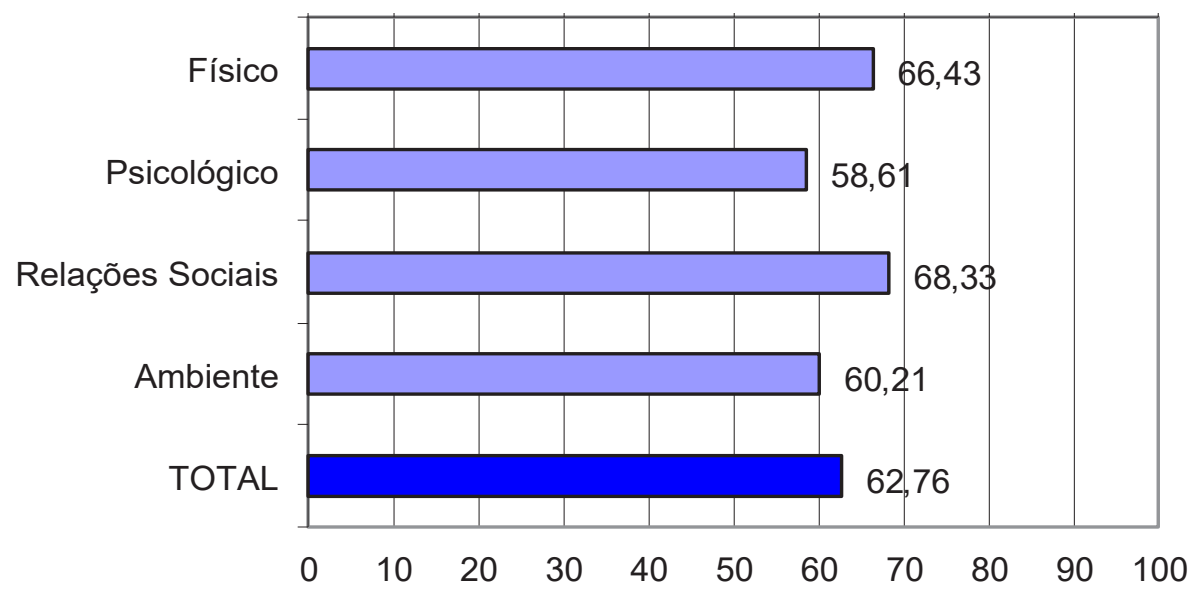

Fonte: Dados da Pesquisa (2019).

As duas primeiras questões do WHOQOL-bref correspondem a uma autoavaliação a respeito da saúde e qualidade de vida das engenheiras, considerando individualmente estas perguntas os resultados demonstram que $53,3 \%$ se encontram satisfeitas com sua saúde e as demais se encontram com um menor 
grau de satisfação. É importante considerar nesta questão a definição de saúde dada pela Organização Mundial da Saúde (OMS) como um estado de perfeito bem-estar físico, mental e social, não sendo apenas a ausência de doença. O questionário não nos permite saber se as respondentes possuíam esse entendimento de saúde como algo além da relação entre paciente e doença, desta forma acredita-se que caso fosse evidenciado no questionário o conceito de saúde dado pela OMS, as respondentes poderiam ter analisado e respondido a questão de forma diferente.

O Gráfico 02 apresenta a média de qualidade de vida de cada faceta. As facetas que apresentaram pior qualidade de vida foram dor de cabeça, dependência de medicação e sentimentos negativos. Os resultados do estudo de Marcacine e colaboradores (2019) apresentam resultados semelhantes, apontando uma associação da dependência de medicamentos a uma má qualidade de vida no domínio psicológico além de ser estaticamente significativo, confirmando os achados desse estudo. A pergunta a respeito do impedimento de realização de atividades por conta de dores físicas apresentou maior grau de variação, dentre as respondentes apenas três afirmaram que era um impedimento pertinente em suas vidas, enquanto as demais disseram passar bem pouco ou nada por esse tipo de incômodo. Quando questionadas se necessitavam de algum tratamento médico para levarem suas vidas diárias, $20 \%$ disseram não precisar de nada, $46,7 \%$ disseram necessitar muito pouco e as demais afirmaram precisar e não descartaram a necessidade do acompanhamento médico; destas, apenas uma engenheira disse carecer extremamente de tratamento médico.

A (Q4) aborda o quanto as engenheiras aproveitam a vida. Essa é uma questão importante, porque a mulher ainda é a maior responsável pelas atividades domésticas e de cuidado com os filhos; essas tarefas atualmente; são somadas com atividades fora do lar e podem causar estresse emocional, sobrecargas físicas e psíquicas (COSTA, 2018). Além disso, por se encontrarem sempre ocupadas com as tarefas do trabalho e da casa, sobra pouco tempo para que essas mulheres participem de atividades cujo único objetivo é o lazer. Pelo fato de não se conhecer exatamente a realidade em que vivem as entrevistadas, como, por exemplo, se elas moram com alguém ou se têm filhos, fica difícil fazer uma reflexão mais aprofundada dessa pergunta, mas o que mostram os dados é que $60 \%$ das engenheiras entrevistadas consideram aproveitar "mais ou menos" suas vidas. Além disso, foi abordado, nos questionários, em que medida as engenheiras têm oportunidades de fazer uma atividade de lazer e apenas $26,7 \%$ responderam tê-las.

Ainda em relação às questões da jornada estendida de trabalho das mulheres, as engenheiras foram questionadas se têm energia suficiente para seu dia a dia, 46,7\% dizem ter uma energia média, 26,7\% afirmam ter muito pouco e $26,7 \%$ responderam ter muita energia. Também se questionou as engenheiras acerca do sono: $33,3 \%$ delas se consideram satisfeitas com seu sono; $26,7 \%$, muito satisfeitas; $26,7 \%$, nem satisfeitas e nem insatisfeitas; e 13,3\%, insatisfeitas.

Buscou-se analisar o rendimento das engenheiras no mercado de trabalho e identificou-se que $60 \%$ consideram que o dinheiro que elas ganham é suficiente para atender às suas necessidades; $20 \%$, muito suficiente; e 3\%, muito pouco ou nada suficiente. A diferença salarial, como discutido acima, ainda é uma das marcas da desigualdade de gênero na atualidade e o fato das mulheres engenheiras não se encontrarem, normalmente, em lugares de liderança (CONFEA, 2020) pode acentuar ainda mais as diferenças salariais e consequentemente trazer insatisfação no trabalho por parte dessas mulheres.

A falta de reconhecimento profissional é um fator que pode gerar desmotivação no trabalho e interferir na autoestima dos colaboradores (GIACOMELLI et al., 2016). Porém, neste estudo, esse fator foi pouco notado, visto que os resultados da avaliação da autoestima das engenheiras apontam um valor consideravelmente bom: de 68,33\%. Além disso, os resultados apresentaram também um bom índice de capacidade de trabalho $(63,33 \%)$.

Portanto, mulheres que ocupam cargos que não são dominados pelo seu gênero podem apresentar um declínio em sua saúde principalmente na área psicológica o que leva a consequências como a má qualidade de vida, sinais e sintomas de futuras patologias; diante disso, é ressaltada a necessidade de mais estudos 
sobre a qualidade de vida em diferentes profissões que as mulheres exercem (MILNER et al., 2018).

Gráfico 02 - Média da qualidade de vida por facetas dos domínios.

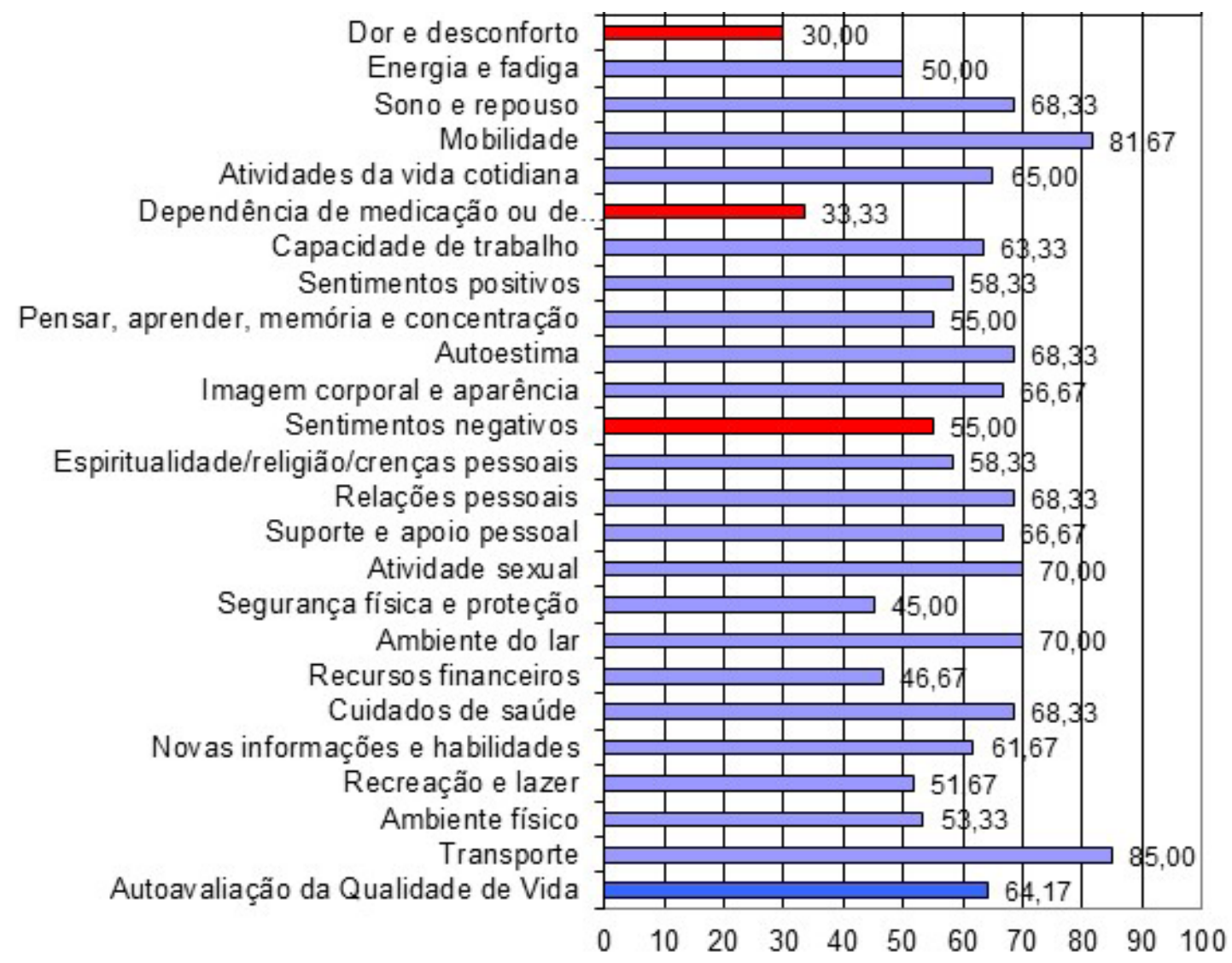

Fonte: Dados da Pesquisa (2019).

\section{CONCLUSÃo}

O estudo permitiu identificar a percepção das engenheiras civis sobre sua qualidade de vida e verificar os domínios que apresentaram menor média, neste caso, o psicológico. Apesar de a hipótese aventada, qual seja, de que as mulheres, por ainda serem minoria em uma área tão masculinizada e dificilmente reconhecidas profissionalmente, como mostra o baixo número de engenheiras em cargos de liderança, teriam sua qualidade de vida comprometida, não tenha se confirmado, visto que as engenheiras apontaram ter uma boa qualidade de vida, é importante considerar que as maiores queixas dadas por essas mulheres se dão no aspecto psicológico.

Sugere-se mais estudos que possam avaliar a qualidade de vida de mulheres na engenharia civil como em outras áreas, para proporcionar mais estratégias para o autocuidado e promoção da saúde para essa população.

\section{Agradecimentos}

Ao Instituto Cesumar de Ciência, Tecnologia e Inovação (ICETI) pela concessão da Bolsa de Produtividade em Pesquisa que possibilitou a realização desse estudo e também ao CREA-SP pelo envio do questionário utilizado nessa pesquisa às engenheiras associadas. 


\section{REFERÊNCIAS}

BRASIL. Ministério da Saúde (MS). Política Nacional de Promoção da Saúde (versão preliminar). Brasília: MS, 2014. 32p.

CERIBELI, H. B.; ROCHA, G. B. S.; PEREIRA, M. R. Mulheres em cargos de chefia: desafios e percepções. Canoas, n. 36, p. 9-24, 2017.

CHAllOUTS, C. U.; ELIAS, M. L. G. G.; SILVA, T. M. G. DA. Desigualdades de Gênero, Campos de Conhecimento e Atuação Profissional de Engenheiras Civis. Revista Cesumar - Ciências Humanas e Sociais Aplicadas, v. 24, n. 2, p. 399-417, 2019.

CONFEA - Conselho Federal de Engenharia e Agronomia. Programa Mulher Sistema CONFEA/ CREA E MÚTUA 2018-2020, Brasília, 2020. Disponível em: http://normativos.confea.org.br/downloads/ anexo/1395-19.pdf2020.

CHIESA, A. M.; ZOBOLI, E. L. C. P.; GRANJA, G. F. Atenção à Saúde na Perspectiva da Equidade. In: PELICIONI, M. C. F.; MIALHE, F. L. (Org.). Educação e promoção da saúde: teoria e prática. 2. ed. Rio de Janeiro: Santos, 2019, p. 289-207.

COSTA, F. A. DA. Mulher, Trabalho e Família: os Impactos do Trabalho na Subjetividade da Mulher e em suas Relações Familiares. Pretextos - Revista da Graduação em Psicologia da PUC Minas, v. 3, n. 6, p. 434-452, 2018.

DIAS, M. S. L. A escolha feminina na área das profissões tecnológicas: impactos na subjetividade. Cadernos de Gênero e Tecnologia, v. 9, n. 33, p. 3-31, 2016.

FARO, A.; PEREIRA, M. E. Raça, racismo e saúde: a desigualdade social da distribuição do estresse. Estudos de psicologia, v. 16, n. 3, p. 271-278, 2011.

FLECK, M. P. DE A. et al. Desenvolvimento da versão em português do instrumento de avaliação de qualidade de vida da OMS (WHOQOL-100). Revista Brasileira de Psiquiatria, v. 21, n. 1, p. 19-28, 1999.

GIACOMELLI, W.; BORGES, G. D. R.; DOS SANTOS, E. G. Determinantes da Desmotivação no Trabalho: uma investigação teórica e empírica. Revista de Administração de Roraima - RARR, v. 6, n. 1, p. 4-17, 2016.

KERGOAT, D. Divisão sexual do trabalho e relações sociais de sexo. In: HIRATA, Helena et al. (Org.). Dicionário crítico do feminismo. 2a ed. São Paulo: UNESP, 2009. p. 67-75.

LAGES, S. R. C. et al. O preconceito racial como determinante social da saúde - a invisibilidade da anemia falciforme. Gerais: Revista Interinstitucional de Psicologia, v. 10, n. 1, p. 109-122, 2017.

LIBERATO, T. F.; ANDRADE, T. H. N. Relações de gênero e inovação: atuação de mulheres nos NITs paulistas. Revista Estudos Feministas, v. 26, n. 2, 2018. 
Challouts et al.

LOMBARDI, M. R. Engenheiras na construção civil: a feminização possível e a discriminação de gênero. Cadernos de Pesquisa, v. 47, n. 163, p. 122-146, 2017.

MAKSUD, I. Estigma e discriminação: desafios da pesquisa e das políticas públicas na área da saúde. Physis: Revista de Saúde Coletiva [online], v. 24, n. 01, p. 311-321, 2014.

MARCACINE, P. R. et al. Qualidade de vida, fatores sociodemográficos e ocupacionais de mulheres trabalhadoras. Ciênc. Saúde Colet., v. 24, n. 3, 2019.

MASSIGNAM, F. M.; BASTOS, J. L. D.; NEDEL, F. B. Discriminação e saúde: um problema de acesso. Epidemiol. Serv. Saúde, v. 24, n. 3, p. 541-544, 2019.

MATOS, M. Gênero. In: FLEURY-TEIXEIRA, E.; MENEGHEL, S. N. (Org.). Dicionário Feminino da Infâmia: Acolhimento e Diagnóstico de Mulheres em Situação de Violência. Rio de Janeiro: Fiocruz, 2015, p. 153-155.

MILNER, A.; KING, T.; LAMONTAGNE, A.; BENTLEY, R.; KAVANAGH, A. Men's work, Women's work, and mental health: A longitudinal investigation of the relationship between the gender composition of occupations and mental health. Soc. Sci. Med., v. 204, p. 16-22, 2018.

OLIVEIRA, M. F.; GONÇALVES, M. C. S.; DIAS, C. M.; ZAGANELLI, M. V. O trabalho das mulheres em áreas relacionadas à tecnologia e engenharia: estudo de caso sobre a inclusão feminina na construção civil. HUMANIDADES \& TECNOLOGIA EM REVISTA (FINOM), v. 22, 2020.

ORGANIZAÇÃO MUNDIAL DA SAÚDE. 1st Global Conference on Health Promotion. Ottawa: OMS, 1986.

PEDROSO, B.; PILATTI, L. A.; REIS, D. C. Cálculo dos escores e estatística descritiva do WHOQOL-bref através do Microsoft Excel. Revista Brasileira de Qualidade de Vida, v. 2, n. 1, p. 31-36, 2010.

PEREIRA, É. F.; TEIXEIRA, C. S.; SANTOS, A. Qualidade de vida: abordagens, conceitos e avaliação. Revista Brasileira Educação Física e Esporte, v. 26, n. 2, p. 241-250, 2012.

PICANÇO, L. S. Amélia e a mulher de verdade: representações dos papéis da mulher e do homem em relação ao trabalho e à vida familiar. In: ARAUJO, C.; SCALON, C. (Orgs.). Gênero, família e trabalho no Brasil. Rio de Janeiro: Editora FGV, 2005, p.149-172.

RODRIGUES, T. F. Desigualdade de Gênero e Saúde: Avaliação de Políticas de Atenção à Saúde da Mulher. Revista Cantareira, Edição 22, p. 203-216, 2015.

SCHWARCZ, L. M. Sobre o autoritarismo brasileiro. São Paulo: Companhia das Letras, 2019. 294p. SCOTT, J. Gênero: uma categoria útil de análise histórica. Revista Educação e Realidade, v. 20, n. 2, p. 71-99, 1995.

SILVA, T. M. G.; BERNUCCI, M. P.; GARCIA, L. F.; SILVA, A. T. C. Violência praticada por parceiros íntimos e saúde: representações de mulheres de um município do Paraná. Revista Saúde, v. 46, n. 1, p. 
$1-12,2020$.

WHOQOL Group. The development of the World Health Organization quality of life assessment instrument (the WHOQOL). In: ORLEY, J.; KUYKEN, W. (Orgs.). Quality of life assessment: international perspectives. Paris: Springer, 1993. p. 41-60. 